\title{
A REPORT ON LECANIDAE (ROTIFERA: MONOGONONTA) FROM ANDHRA PRADESH, INDIA, INCLUDING SIX NEW DISTRIBUTION RECORDS WITH NOTES ON THEIR CONTEMPORARY TAXONOMIC NOMENCLATURE
}

\author{
S.Z. Siddiqi ${ }^{1} \&$ M. Karuthapandi ${ }^{2}$
}

1,2 Zoological Survey of India, Freshwater Biological Regional Centre, Hyderabad, Andhra Pradesh 500048, India ${ }^{1}$ szsiddiqi_hyd@yahoo.co.in (corresponding author), ${ }^{2}$ kpandi83@gmail.com

\begin{abstract}
The Lecane-species complex taxonomy the world over, witnessed a state of flux, causing confusion and controversies, among world's taxonomists over the treatment of various subgenera, taxa and sub and or infraspecific categories of the species rich genus Lecane Nitzsch 1827, on the basis of structure/shape of key, morphological features like foot/toes, lorica, etc. The taxonomic scenario in India, relying heavily on the classical, divergent taxonomic approaches presented a picture of more chaos/confusion, following poor accessibility to contemporary revisionary studies until the recent past. Despite revisionary studies across the world, a few notable Indian studies continued to be burdened with old nomenclature. This short communication reports for the first time ever, 33 valid species of lecanid rotifers (Lecanidae), including six new distributional records from Greater Hyderabad region and the entire state of Andhra Pradesh too with comments on their current nomenclature. Further, limnobiological correlation between five physicochemical parameters and rotifer associations revealed, L. bulla, L. closterocerca, L. hamata, L. ludwigi, L. luna and L. papuana as euryokous species, showing tolerance to a wide range of abiotic factors and habitats too.
\end{abstract}

Keywords: Euryokous, morphological variant, Lecanid taxonomy, limnological indicator and taxa.

Taxonomy of rotifers, the world over, in particular lecanid rotifers too, continued to remain in a state of flux in view of high species diversity, plasticity, regional and /or seasonal variability and geographic endemicity. warranting global revisionary studies on many groups/ families. In fact the taxonomy of ubiquitous, biogeographically significant Lecane species-complex was in a state of flux for decades, and indeed a major irritant to taxonomists world wide, following differences, until the mid nineties; Segers (1995) finally setting to rest all prevailing confusions/ controversies, assigning all species under a single genus Lecane Nitzsch, 1827. The Indian scenario on lecanid taxonomy was no different and the confusion continued to prevail until the beginning of the century, ironically enough for lack of accessibility to standard world revisionary studies/literature and serious comprehensive Indian studies on the groups, despite its abundance and distinct tropical characteristics (Sharma 1996).

Notable pioneering taxonomic studies on lecanid rotifer from Andhra Pradesh were initiated by Dhanapathi (1976a) recording two new species, Lecane bidentata and $L$. donnerianus, including five first distribution reports. Incidentally, soon he also reported an other new taxon, Lecane easwari, from West Godavari and Krishna districts, Andhra Pradesh Dhanapathi (1976b). About the same time, Rao \& Mohan (1977) reported Monostyla obtusa Murray, 1913, from Municipal tank, Mudasarlova, Visakhapatanam, and later seven new

DOI: http://dx.doi.org/10.11609/JoTT.03095.4556-61 | ZooBank: urn:Isid:zoobank.org:pub:5888AD85-A94A-46F8-B578-CB9CF3C89BB0

Manuscript details: Ms \# 03095 | Received 11 February 2012 | Final received 28 June 2013 | Finally accepted 02 July 2013

Citation: Siddiqi, S.Z. \& M. Karuthapandi (2013). A report on Lecanidae (Rotifera: Monogononta) from Andhra Pradesh, India, including six new distribution records with notes on their contemporary taxonomic nomenclature. Journal of Threatened Taxa 5(11): 4556-4561; http://dx.doi.org/10.11609/JoTT.o3095.4556-61

Copyright: @ Siddiqi \& Karuthapandi 2013. Creative Commons Attribution 3.0 Unported License. JoTT allows unrestricted use of this article in any medium, reproduction and distribution by providing adequate credit to the authors and the source of publication.

Funding: The junior author (MK) is supported by Research Fellowship (SRF) through ZSI by MoEF, New Delhi.

Competing Interest: None.

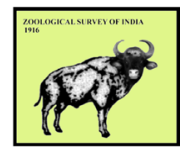

Acknowledgements: The authors are grateful to Dr. K. Venkatraman, Scientist G and Director, Zoological Survey of India, Kolkata for encouragement, infrastructural facilities, fellowship (KP) and to fellow scientists/staffs for cooperation. 
distribution records (Rao \& Mohan 1982). Arshaduddin \& Khan (1991) reported 40 species of rotifers, including 10 species under the genus Lecane Nitzsch, 1827 from organically polluted temporary seasonal ponds from Osmania University, Jamia Osmania campus, Hyderabad, assigning them saprobic values following Sladecek (1983). Surprisingly enough, there are no comprehensive taxonomic studies on the family Lecanidae from the state, indeed Indian region too, save one by Sharma (1996) reporting 74 species of the genus Lecane from India. The actual number, including infraspecific varieties, may be marginally higher, going by recently published reports (Sharma 2007; Sharma \& Sharma 2010, 2011). This short communication reports 20 species from Greater Hyderabad region, and the state, upgrading the tally to 33 species of lecanid rotifers, including six new distribution records from the state of Andhra Pradesh.

\section{Material and Methods}

A brief description of the three study sites follows. Site 1 , a masonry pond (hereafter designated as MP), $17^{0} 21^{\prime} 28^{\prime \prime} N$ \& $78^{\circ} 23^{\prime} 32^{\prime \prime} E$, area $200 m^{2}$, rain fed, walled on three sides, a natural depression in undulating terrain, located at Attapur, Rajendranagar, Hyderabad, was chosen for its proximity, size and considerable (rainwater) retention period, and habitat variability. Site 2 Landscape Garden Pond, Osmania University, Hyderabad, abbreviated as LGP, $17^{\circ} 25^{\prime} 02^{\prime \prime} \mathrm{N}$ and $78^{\circ} 31^{\prime} 52^{\prime \prime} \mathrm{E}$, rain fed, roughly square-shaped, natural depression, in undulating terrain, has an area $\left(0.44 \mathrm{~km}^{2}\right)$, remains dry for over 8-9 months, save during the southwest monsoons (July-September 2011) and a month or two beyond. The site was chosen as previous studies recorded rich rotifer diversity and therefore served as a comparable study. Site 3 Bandakum cheruvu, Bandakum Village, Pattancheruvu, Medak, shortened as BP, $17^{0} 30^{\prime} 44^{\prime \prime} \mathrm{N} \& 78^{\circ} 19^{\prime} 11^{\prime \prime} \mathrm{E}$, located in mildly undulating terrain, is a shallow habitat, depth (1.5-2 m), area $1.58 \mathrm{~km}^{2}$, receives inflows from precipitation and a mild stream located upwards. Of the three pond habitats, the masonry pond has a higher level of water retention period, following rock-cemented boundaries and no free out flows, high macrophyte infestations albeit low water levels until January 2012. There are no significant geomorphic differences in the three ponds but were chosen for proximity, accessibility and academic necessity (species richness, vis-à-vis short life cycle) and potential for aquatic ecological research.

The physical factors like temperature, $\mathrm{pH}$, electrical conductivity and total dissolved solids were estimated in situ with the help of electronic dip testers (Hanna make
pH ep, $\operatorname{dip} C$, dip D, etc.) while samples for dissolved oxygen (200ml BOD, glass bottles) were Winklerized in the field. Surface water samples were collected in wide mouth PVC containers (Tarsons bottles with plugs and screw cap) for estimations of various physicochemical parameters. The dissolved oxygen was analyzed titrimetrically, while total alkalinity (OR-AIK-01), hardness (OR-TH-01), chloride (OR-Cl-01), calcium (OR-Ca-01), magnesium and inorganic plant nutrients - orthophosphate (P-PO, OR-P-01) nitrogen-nitrate $(\mathrm{N}$ $\mathrm{NO}_{2}$, OR-NO $\left.-\mathrm{O} 1\right)$, nitrogen - nitrite $\left(\mathrm{N}-\mathrm{NO}_{3}, \mathrm{OR}-\mathrm{NO}_{3}-\mathrm{O} 1\right)$ and ammonical nitrogen ( $\left.\mathrm{N}-\mathrm{NH}_{3}, \mathrm{OR}-\mathrm{NH}_{3}-\mathrm{O} 1\right)$ analyzed using Orlab Instruments Pvt. Ltd. field kits, following standard limnological works/texts (Lind 1979; Wetzel $\&$ Likens 2000). Plankton samples from surface waters (littoral region) were collected by towing long handled plankton net (No 25. 64 $\mu \mathrm{m}$ mesh size) and wading through waist deep shallow water, where feasible. $100 \mathrm{ml}$ plankton sample in PVC containers, preserved in $4 \%$ neutralized formalin, (Registration numbers FBRC/ZSI/ UN: 6079-6714), stained in eosin ink, manually sorted and observed under a Nikon 80i light microscope and digitally photographed. Standard regional taxonomic works (Patil \& Gouder 1989; Battish 1992; Dhanapathi 2000) and others were used for confirming taxonomic identity and contemporary status (Segers 1995 \& 2007).

\section{Results and Discussions}

The present study revealed high species richness in the genus Lecane, 20 species to be precise, including six new distributional records from the region. Lecane species like L. aculeata (Jakubski, 1912), L. haliclysta Harring \& Myers, 1926, L. furcata (Murray, 1913), L. pawlowskii Wulfert, 1966, L. pyriformis (Daday, 1905), and L. ruttneri (Hauer, 1938) form new distribution records from the region (Images 1-6). It further documents the range of values of physicochemical parameters and ecological conditions of the ponds, reiterating the ecological importance of the littoral regions and its continued under estimation in Indian studies, despite its faunistic richness and biogeographic significance (Segers et al. 1994). An exhaustive updated list of valid species of highly plastic, species-rich genus Lecane recorded from Andhra Pradesh, including new taxa and infraspecific varieties recorded to date, and their present taxonomic status is appended, for the benefit of Indian researchers (after Segers 1995, 2007).

The two new taxa reported by Dhanapathi (1976a) viz. Lecane bidentata and Lecane donnerianus are now indeed junior synonyms of L. batillifer (Murray, 1913) and L. donneri Chengalath \& Mulamoottil, 1974; Segers 
(2007) adding L. bidentata as species enquirendae. Further, of the five new distribution records reported then (Dhanapathy 1976), Monostyla styrax Harring \& Myers, 1926 is subsumed as L. styrax (Harring \& Myers, 1926 ) and M. tethis is a synonym of L. furcata (Murray, 1913). While Monostyla obtusa Murray, 1913, reported by Rao \& Mohan (1977) is Lecane obtusa (Murray, 1913), the current taxonomic replacements of some of the other seven (Rao \& Mohan 1982) are as under: Lecane acanthinula (Hauer, 1938) (=L. acronycha Harring \& Myers, 1926), L. curvicornis (Murray, 1913) $(=L$. curvicornis var. padespares (Arora, 1963), L. bulla (Gosse, 1851) [=Monostyla bulla (Gosse, 1851)], L. closterocerca (Schmarda, 1859) [=Monostyla closterocerca Schmarda, 1859], L. lunaris Ehrenberg, 1832 [= Monostyla lunaris Ehrenberg, 1832] and L. unguitata (Fadeev, 1925) [=Monostyla unguitata ((Fadeev, 1925)] Lecane ploensis (Voigt, 1902) is now a junior synonym of L. pideis (Harring \& Myers, 1926) and L. ohioensis Herrick, 1885 has been subsumed by L. obtusa (Murray, 1913). (Table 1).

The short duration seasonal studies revealed 16 (MP), 09 (LGP), and 15 spp. (BGP) respectively, of genus Lecane with masonry pond collections exhibiting relatively high species richness (18 spp.), perhaps more, following relatively higher habitat heterogeneity, nutrient loading, from surface runoffs, and a higher undisturbed retention period. The LGP, Osmania University, is an open system, having a slopy terrain all around that facilitates substantial outflows, and consequently relatively shorter retention period and habitat homogeneity. Further, while the BG pond retains water for the most part, it too has habitat homogeneity. The MP, Attapur, littered with boulders, higher depth $(1-1.5 \mathrm{~m})$ and littoral macrophytes, has higher aquatic productivity, witnessed through production of fish nekton [Channa punctatus (Perciformis: Channidae) - Snake heads], appear around the third month October], and offers more ecological niches. Lecane bulla (O.F. Muller, 1776), one of the highly

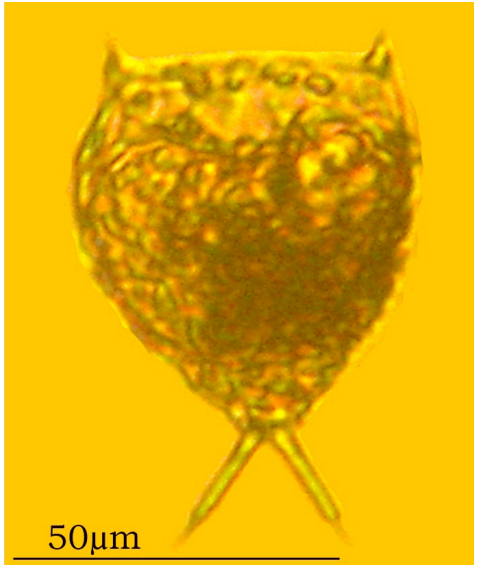

Image 1. Lecane aculeata (Jakubski, 1912)

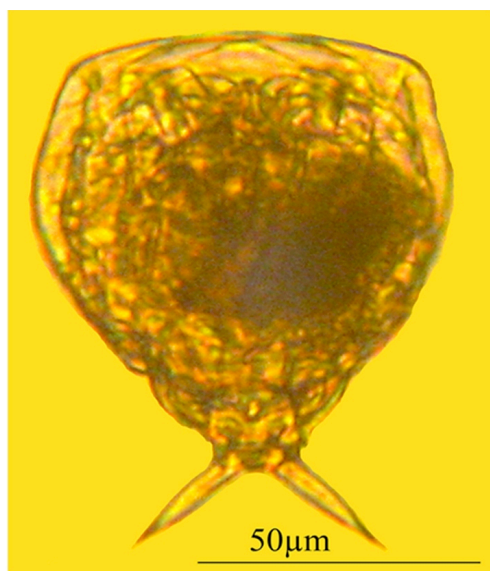

Image 4. Lecane ruttneri (Hauer, 1938)

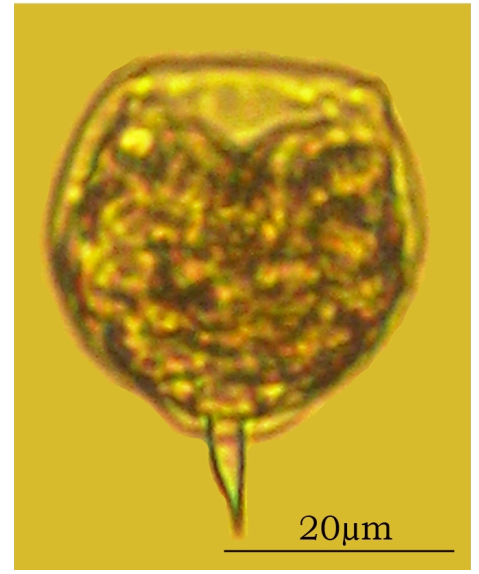

Image 2. Lecane pyriformis (Daday, 1905)

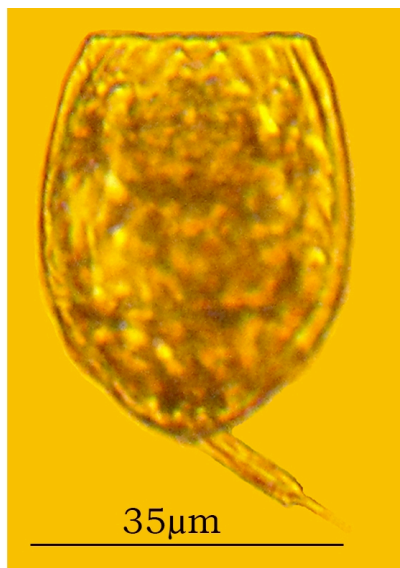

Image 5. Lecane furcata (Murray, 1913)

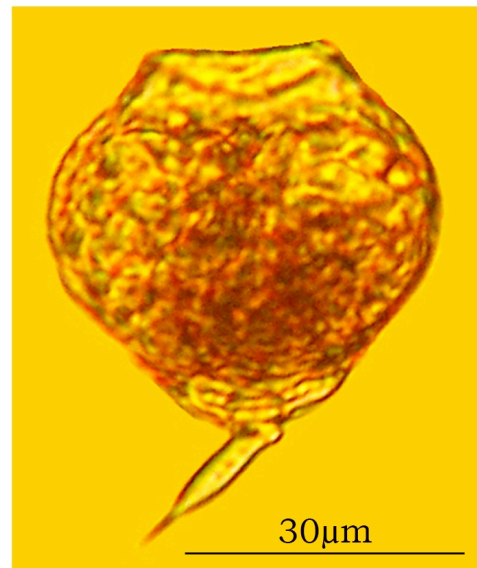

Image 3. Lecane pawlowskii Wulfert, 1966

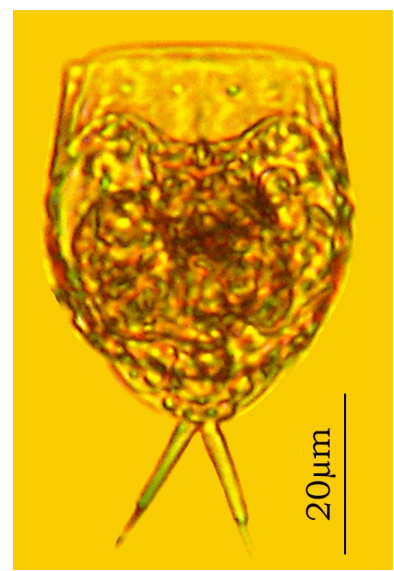

Image 6. Lecane haliclysta Harring \& Myers, 1926 
morphologically variable taxon, was observed to be numerically abundant, inhabiting all ponds, all through the study, while L. pyriformis and L. quadridentata were common in LGP, Osmania University, Hyderabad. Further, of the 22 spp. recorded, eight species - L. bulla, L. closterocerca, L. hamata, L. ludwigi, L. luna, L. lunaris and $L$. papuana-occurred commonly in all three ponds. Sorensen's index/similarity coefficients (1948) were used to evaluate percent similarity between the three pond ecosystems. The quotient of similarity (QS) worked out to 32,31 and $32 \%$, respectively. Further the similarity/ dissimilarity index between the three worked out to 36 and $64 \%$, respectively.

Table 2 highlights the range of values of various physicochemical parameters in littoral waters, besides range of values for inorganic plant nutrient-various forms of phosphorous ( $\left.\mathrm{P}-\mathrm{PO}_{4} \mathrm{mg} / \mathrm{L}\right)$ and Nitrogen $\left(\mathrm{N}-\mathrm{NO}_{2}, \mathrm{~N}-\mathrm{NO}_{3}\right.$ and $\left.\mathrm{N}-\mathrm{NH}_{3} \mathrm{mg} / \mathrm{L}\right)$. Despite ecological potential of rotifers as possible limnological indicator, no categorical correlation can be drawn between rotifer fauna and the wide range of physicochemical indicators especially temperature, hydrogen ion concentration $(\mathrm{pH})$, alkalinity, chlorides and or conductivity, in view of wide environmental tolerance range of the various species complexes, following difference in not just morphology but, genetics too (Kuczynski 1987). Present study also at best are indicative of range of tolerance of various Lecane species to wide $\mathrm{pH}$ (7.1-8.0), high chloride (61-506 $\mathrm{mg} / \mathrm{l})$, high alkalinity $(128-331 \mathrm{mg} / \mathrm{l})$ and lower medium conductivity (0.8-1.89 mS). Co-incidentally enough, six of the species namely $L$. bulla, $L$. closterocerca, $L$. hamata, L. ludwigi, L. luna and L. papuana are common to all studies from the region. Going by limnological indicators and rotifer associations (Kuczynski 1987), they may be categorized as euryokous.

\section{REFERENCES}

Arshaduddin, M. \& M.A. Khan (1991). Rotifer Fauna of some seasona ponds of Osmania University Campus, Hyderabad (Andhra Pradesh) India. Indian Journal of Microbial Ecology 2: 29-40.

Battish, S.K. (1992). Freshwater Zooplankton of India. Oxford and IBH Publishing Company Private Limited, Calcutta, vi+233pp.
Dhanapathi, M.V.S.S.S. (1976a). Rotifers from Andhra Pradesh, India -III. Family Lecanidae including two new species. Hydrobiologia 48 (1): 9-16.

Dhanapathi, M.V.S.S.S. (1976b). A new Lecanid rotifer from India. Hydrobiologia 50: 191-192.

Dhanapathi, M.V.S.S.S. (2000). Taxonomic notes on the Rotifers from India (from 1889-2000). Indian Association of Aquatic biologists (IAAB), publication No. 10. Hyderabad, 1-180pp.

Kuczynski, D. (1987). The rotifer fauna of Argentina Patagonia as a Potential limnological indicator. Hydrobiologia 150: 3-10.

Lind, O.T. (1979). Hand Book of Common Methods in Limnology (2 ${ }^{\text {nd }}$ Edition). The C.V. Mosby Company, St. Louis, Toronto, London, $199 p$.

Patil, C.S. \& B.Y.M. Gouder (1989). Freshwater Invertebrates of Dharwad (Karnataka State India). Prasaranga, Karnataka University, Dharwad, ix+144pp.

Rao, R.K. \& P.C. Mohan (1977). Monostyla obtusa Murray (Rotifer Lecanindae) - A New Record from India. Geobios new Reports 4(3): 188.

Rao, R.K. \& P.C. Mohan (1982). Studies on Freshwater Rotifer from Visakhapatnam, India. Records of the Zoological Survey of India 79: 397-403.

Segers, H. (1995). Rotifera 2: The Lecanidae (Monogononta). In: Dumont H.J. \& T. Nogrady (eds.) Guides to Identification of Micoinvertebrates of the Continental Waters of the World. SPB Academic Publishing bv. Amsterdam, Netherlands, 226pp.

Segers, H. (2007). Annotated checklist of the rotifers (Phylum Rotifera), with notes on nomenclature, taxonomy and distribution. Zootaxa 1564: 1-104.

Segers, H., S.S.S. Sarma, F.K. Kakkassery \& C.K.G. Nayar (1994). New records of Rotifera from India. Hydrobiologia 287(3): 251-258.

Sharma, B.K. (1996). Biodiversity of freshwater rotifera India - A status report. Proceedings of the Zoological Society 49(2): 73-85.

Sharma, B.K. (2007). Notes on rare and interesting rotifers (Rotifera: Eurotatoria) from Loktak lake, Manipur - a Ramsar Site. Zoo's Print. Journal 22(9): 2816-2820; http://dx.doi.org/10.11609/JoTT. ZPJ.1742.2816-20

Sharma, B.K. (2009). Diversity of Rotifers (Rotifera: Eurotatoria) of Loktak Lake, Manipur, north-eastern India. Tropical Ecology 50(2): 277-283

Sharma, B.K. \& S. Sharma (2010). Notes on new records of monogononta Rotifers (Rotifera: Eurotatoria) from Flood plain Lakes of Assam. Records of the Zoological Survey of India 110 (Part2): 49-55.

Sharma, B.K. \& S. Sharma (2011). Deepor Beel revised: new records (Rotifera: Eurotatoria) with remarks on interesting species. Journal of Threatened Taxa 3(1): 1437-1444; http://dx.doi.org/10.11609/ JoTT.o2482.1437-44

Sladecek, V. (1983). Rotifers as indicators of water quality. Hydrobiologia 100: 169-201.

Sorensen, T. (1948). A method of establishing groups of equal amplitude in plant sociology based on similarity of species and its application to analyses of the vegetation on Danish commons. Biologiske Skrifter/Kongelige Danske Videnskabernes Selskab 5(4): $1-34$

Wetzel, R.G \& G.E. Likens (2000). Limnological Analyses-3 $3^{\text {rd }}$ Edition. Springer (India) Private limited, New Delhi, India, xvi+426pp. 
Table. 1 List of Lecane species reported from Hyderabad, Andhra Pradesh, India. (Dhanapathi 1976 onwards). Asterisk (*) as superscripts against the taxon name indicates first geographic records, while + (plus) sign indicates occurrence and - (Minus) as nonoccurrence.

\begin{tabular}{|c|c|c|c|c|c|}
\hline & \multirow[b]{2}{*}{$\begin{array}{l}\text { Rotifera } \\
\text { Lecanidae Remane, } 1933 \\
\text { Lecane Nitzsch, } 1827\end{array}$} & \multicolumn{3}{|c|}{ Temporary freshwater habitats } & \multirow[b]{2}{*}{ Previous studies } \\
\hline & & $\begin{array}{l}\text { Masonry } \\
\text { pond, } \\
\text { Attapur, } \\
\text { Hyderabad }\end{array}$ & $\begin{array}{c}\text { Landscape } \\
\text { Garden pond, } \\
\text { Osmania Univ. } \\
\text { Hyderabad } \\
\end{array}$ & $\begin{array}{c}\text { Bandakum } \\
\text { cheruvu, } \\
\text { Medak District }\end{array}$ & \\
\hline 1 & $\begin{array}{l}\text { Lecane acanthinula (Hauer, 1938) } \\
\text { [=Lecane acronycha Harring \& Myers, 1926] }\end{array}$ & + & - & - & Rao \& Mohan 1982 \\
\hline 2 & L. aculeate (Jakubski, 1912)* & + & - & - & Present study \\
\hline 3 & $\begin{array}{l}\text { L. batillifer (Murray, 1913) } \\
=\text { L. bidentata Dhanapathi, } 1976 \text { sp. enqr. }\end{array}$ & - & - & - & Dhanapathi 1976 \\
\hline 4 & L. bulla (Gosse, 1851) & + & + & + & $\begin{array}{l}\text { Dhanapathi 1976, Rao \& Mohan 1982, } \\
\text { Arshaduddin \& Khan } 1991\end{array}$ \\
\hline 5 & L. closterocerca (Schmarda, 1859) & + & + & + & Dhanapathi 1991, Rao \& Mohan 1982 \\
\hline 6 & $\begin{array}{l}\text { L. crepida Harring, } 1914 \\
\text { [=L.crepida var bengalensis Sharma 1978. Syn. } \\
\text { Lecane vasisthi Sharma, 1980. Sym.] }\end{array}$ & & & & Rao \& Mohan 1982 \\
\hline 7 & $\begin{array}{l}\text { L. curvicornis (Murray 1913) } \\
\text { [=L. curvicornis var. padespares Arora, 1965. Syn.] }\end{array}$ & + & - & + & $\begin{array}{l}\text { Dhanapathi 1976, } \\
\text { Rao \& Mohan } 1982\end{array}$ \\
\hline 8 & $\begin{array}{l}\text { L. donneri Chengalath \& Mulamoottil, } 1974 \\
=\text { L. donnerianus Dhanapathi, } 1976\end{array}$ & - & - & - & Dhanapathi 1976 \\
\hline 9 & L. eswari, Dhanapathi, 1976 & - & - & - & Dhanapathi 1976 \\
\hline 10 & L. furcata (Murray, 1913)* & + & - & - & Present study \\
\hline 11 & L. haliclysta Harring and Myers, 1926* & + & - & - & Present study \\
\hline 12 & L. hamata (Stokes, 1896) & + & + & + & Dhanapathi 1976 \\
\hline 13 & L. hastata (Murray, 1913) & - & - & - & Arshaduddin \& Khan 1991 \\
\hline 14 & L. hornemanni (Ehrenberg, 1881) & - & - & - & Dhanapathi 1976 \\
\hline 15 & L. inopinata Harring and Myers, 1926 & - & - & - & Dhanapathi 1976 \\
\hline 16 & L. lauterborni (Hauer, 1924) & - & - & - & Dhanapathi 1976 \\
\hline 17 & L. leontina (Turner, 1892) & + & + & + & Dhanapathi 1976 \\
\hline 18 & L. ludwigii (Eckstein, 1883) & + & + & + & $\begin{array}{l}\text { Dhanapathi 1976, Rao \& Mohan 1982, } \\
\text { Arshaduddin \& Khan } 1991\end{array}$ \\
\hline 19 & L. Iuna (O. F Muller, 1776) & + & + & + & $\begin{array}{l}\text { Dhanapathi } 1976 \text { \& } \\
\text { Arshaduddin \& Khan } 1991\end{array}$ \\
\hline 20 & $\begin{array}{l}\text { L. lunaris (Ehrenberg, 1832) } \\
\text { [=Monostyla lunaris Ehrenberg, 1832] }\end{array}$ & + & + & + & Dhanapathi 1991, Rao \& Mohan 1982 \\
\hline 21 & $\begin{array}{l}\text { L. obtusa (Murray, 1913) } \\
\text { [=Monostyla obtusa (Murray, 1913) } \\
=\text { L. ohioensis (Herrick,1885)] }\end{array}$ & - & - & - & $\begin{array}{l}\text { Rao \& Mohan 1982, Arshaduddin \& } \\
\text { Khan } 1991\end{array}$ \\
\hline 22 & L. pawlowskii Walfert, 1966* & + & & & Present study \\
\hline 23 & L. papuana (Murray, 1913) & + & + & + & $\begin{array}{l}\text { Dhanapathi 1976, Rao \& Mohan 1982, } \\
\text { Arshaduddin \& Khan } 1991\end{array}$ \\
\hline 24 & $\begin{array}{l}\text { L. pideis (Harring \& Mayers, 1926) } \\
=\text { L. ploensis (Voigt, 1902) }\end{array}$ & - & - & - & Arshaduddin \& Khan 1991 \\
\hline 25 & L. pyriformis (Daday, 1905)* & - & - & + & Present study \\
\hline 26 & L. quadridentata (Ehrenberg, 1832) & - & + & + & Dhanapathi 1976 \\
\hline 27 & L. ruttneri (Hauer, 1938)* & + & - & + & Present study \\
\hline 28 & L. stenroosi (Meissner, 1908) & - & - & + & Dhanapathi 1976 \\
\hline 29 & $\begin{array}{l}\text { L. stichoclysta Segers, } 1993 \\
\text { [= L. styrax Harring \& Mayers, 1926] }\end{array}$ & - & - & - & Dhanapathi 1976 \\
\hline 30 & $\begin{array}{l}\text { Lecane tenuiseta Harring, } 1914 \\
\text { [=L. tesselata Arora, } 1965 \\
=\text { L. tethis Harring \& Mayers, 1926] }\end{array}$ & - & - & - & Dhanapathi 1976, Rao \& Mohan 1982 \\
\hline 31 & L. tryphema Harring and Myers, 1926 & & & & Rao \& Mohan 1982 \\
\hline 32 & L. ungulata (Gosse, 1887) & + & - & + & Dhanapathi 1976 \\
\hline \multirow[t]{2}{*}{33} & $\begin{array}{l}\text { L. unguitata (Fadeev, 1925) } \\
\text { [=Monostyla unguitata Fadeev, 1925] }\end{array}$ & - & - & + & Rao \& Mohan 1982 \\
\hline & Total Number of Species & 16 & 9 & 15 & \\
\hline
\end{tabular}


Table 2. Range of values different physicochemical parameters, in littoral surface, in three different temporary water habitats including various forms of orthophosphates and ions.

\begin{tabular}{|c|c|c|c|}
\hline Parameters & $\begin{array}{c}\text { Bandakum cheruvu, } \\
\text { Bandakum Village, } \\
\text { Patancheruvu, Medak }\end{array}$ & $\begin{array}{l}\text { Masonry pond, Attapur, } \\
\text { Rajedranagar, Hyderabad }\end{array}$ & $\begin{array}{c}\text { Landscape Garden pond, } \\
\text { Osmania University, } \\
\text { Hyderabad }\end{array}$ \\
\hline Ambient atmospheric temperature ${ }^{\circ} \mathrm{C}$ & $25-33$ & $25-29$ & $27-28$ \\
\hline Sub Surface water temperature ${ }^{\circ} \mathrm{C}$ & $19-29$ & $23-26$ & 27 \\
\hline Light( Lux) & $240-450$ & $240-360$ & 420 \\
\hline${ }_{p} \mathrm{H}$ & $7.5-8.9$ & $7.1-7.7$ & $8.3-8.4$ \\
\hline Electrical conductivity (mS) & $0.97-1.89$ & $0.8-2.2$ & $0.55-0.60$ \\
\hline Total Dissolved Solids (ppm) & $690-1400$ & $880-1155$ & $440-450$ \\
\hline Dissolved Oxygen (mg/L) & $4.6-9.7$ & $4.1-10.3$ & $8.0-8.4$ \\
\hline Total Hardness, $\mathrm{CaCO}_{3}(\mathrm{mg} / \mathrm{L})$ & $189-284$ & $242-406$ & $141-166$ \\
\hline Total Alkalinity, $\mathrm{CaCO}_{3}(\mathrm{mg} / \mathrm{L})$ & 204-331 & $164-208$ & $128-153$ \\
\hline Chlorides, $\mathrm{Cl}-(\mathrm{mg} / \mathrm{L})$ & $182-506$ & $152-248$ & $61-82$ \\
\hline Calcium, $\mathrm{Ca}^{++}(\mathrm{mg} / \mathrm{L})$ & $28-47$ & $52-142$ & $28-38$ \\
\hline Magnesium, $\mathrm{Mg}^{++}(\mathrm{mg} / \mathrm{L})$ & $35-60$ & $31-66$ & $36-41$ \\
\hline $\begin{array}{l}\text { Nutrients } \\
\text { Total Phosphates, } \mathrm{P}-\mathrm{PO}_{\triangle}(\mathrm{mg} / \mathrm{L})\end{array}$ & $0.08-0.4$ & $0.4-0.7$ & $0.09-0.12$ \\
\hline Nitrates, $\mathrm{N}-\mathrm{NO}_{2}(\mathrm{mg} / \mathrm{L})$ & $1-40$ & $1-37$ & $1-1.5$ \\
\hline Nitrites, $\mathrm{N}-\mathrm{NO}_{3}(\mathrm{mg} / \mathrm{L})$ & $0-0.17$ & $0.01-0.4$ & $0.031-0.035$ \\
\hline Ammonia, $\mathrm{N}-\mathrm{NH}_{3}(\mathrm{mg} / \mathrm{L})$ & $0.12-3.66$ & $0.01-0.6$ & - (not determined) \\
\hline
\end{tabular}

